\title{
EL CONTROL INTERNO Y LA COMPETITIVIDAD EN LA EMPRESA ELÉCTRICA REGIONAL DEL SUR S.A. DE ECUADOR
}

\section{INTERNAL CONTROL AND COMPETITIVENESS IN THE EMPRESA ELÉC- TRICA REGIONAL DEL SUR S.A. FROM ECUADOR}

\author{
Judith María Morales Espinosa \\ Magister en Gerencia Contable Financiera -Universidad Nacional de Loja/ Loja - Ecuador. \\ Email: jmme_23@hotmail.com(Autor Corresponsal)
}

[Recibido: 02/05/2016 Aceptado: 14/08/2017]

\section{RESUMEN}

La tesis titulada "Control Interno y Competitividad en la Empresa Eléctrica Regional del Sur" S.A., Ecuador, período 2012-2014", permitió afrontar los problemas encontrados en la empresa, en relación con la falta de control en el sector de la Gestión Operativa. Con el fin de ser competitivos y cumplir con s us o bligaciones como un buen s ervicio al cliente, hemos aplicado la metodología de la investigación científica. Hemos utilizado la encuesta como una técnica para recopilar información directamente del público y de los trabajadores, así como de expertos profesionales que tienen una relación directa o indirecta con la empresa. La tesis se desarrolló con la recopilación de información y datos de diferentes especialistas que contribuyeron a consolidar el desarrollo de las variables: Problemas de implementación de control interno y competitividad en EERSSA, ubicada en la Ciudad de Loja en Ecuador. La técnica de encuesta con doce (12) preguntas permitió obtener datos. La información permitió la tabulación y trabajo en la formulación gráfica, con sus respectivas interpretaciones; La hipótesis se llevó a cabo para determinar las conclusiones y recomendaciones. Finalmente, se determinó que la implementación del control interno influye positivamente en la rentabilidad de la empresa.

\section{PALABRAS CLAVE}

Control Interno, competitividad, rentabilidad.

\begin{abstract}
The thesis titled "Internal Control and Competitiveness in Empresa Eléctrica Regional del Sur" S.A., Ecuador, period 2012-2014", allowed to meet the problems found in the company, concerning to the lack of control in the Operational Management sector. In order to be competitive and fulfill its obligations such as a good Customer Service, we have applied the methodology of scientific investigation. We have used the survey as a technique for collecting information directly from public and workers as well as professional experts who have a direct or indirect relationship with the company. The thesis was developed with the collection of information and data from different specialists, who contributed to consolidate the development of the variables: Problems regarding to implementation of internal control, and competitiveness in EERSSA, located in the City of Loja in Ecuador. The technique of survey with twelve (12) questions allowed to obtaining data. The information allowed the tabulation and work in the graphic formulation, with their respective interpretations; the hypothesis was carried out to determine the conclusions and recommendations. Finally, it was determined that the implementation of internal control positively influences the profitability of the company.
\end{abstract}

\section{KEYWORDS}

Internal control, competitiveness, profitability.

Como Citar: Morales, J. (2017). El control interno y la competitividad en la empresa Eléctrica Regional del sur S.A. de Ecuador - periodo 2012-2014. Quipukamayoc, 25(49), 67-72. doi: 


\section{INTRODUCCIÓN}

Siendo la energía eléctrica el motor y eje fundamental para el desarrollo del país y coadyuvante principal del mejoramiento de la calidad de vida del ser humano, el Consejo Nacional de Electricidad (CONELEC) pone a disposición de todas las instituciones y personas vinculadas con la actividad del sector eléctrico. En procura de mejorar las condiciones de servicio a los usuarios, ha sufrido varios cambios, que buscan facilitar el desarrollo de este Sector.

El CONELEC aspira que este servicio constituya un medio de progreso sobre el desenvolvimiento del sector eléctrico ecuatoriano, especialmente, para quienes están vinculados con esta importante área de desarrollo del país.

La Empresa Eléctrica Regional del Sur S.A brinda uno de los servicios básicos más importantes como es generar, distribuir, comercializar y otorgar el servicio de energía eléctrica, a los clientes de las provincias de Loja, Zamora Chinchipe y Morona Santiago que constituye en un aporte significativo en la economía del Ecuador, la EERSSA. Sin embargo, la deficiente gestión de los recursos económicos no ha permitido lograr elevar la calidad confiabilidad y seguridad del nivel de vida y el desarrollo del beneficio a los clientes, al contar con un servicio óptimo y cumplir con el "Plan Nacional del Buen Vivir".

Concomitantemente, los informes de Auditoría Externa y Comisario incluyen resultados de evaluación del control interno con recomendaciones incoherentes sin un análisis profundo de sus componentes para asegurar su solidez administrativa y financiera, proyectando la asesoría a la Gerencia de las Empresas con tendencia al eficiente manejo de los recursos económicos y el logro de los objetivos institucionales.

Para este trabajo, elegimos la perspectiva filosófica por su radicalidad e implicaciones éticas, trasladables a la evaluación de la aplicación del control interno en la competitividad de la Empresa Eléctrica Regional del Sur S.A. del Ecuador.

De las consultas realizadas a bibliotecas de Escuelas de Postgrado de la Facultades de Ciencias Contables, Económicas y Financieras de Universidades de Lima Metropolitana, así como de otros países he logrado identificar trabajos de Investigación realizados, que guardan cierta relación con mi investigación, por lo que nos permitimos mencionar a los siguientes:

Gómez (2001) destaca que la evaluación del control interno define las fortalezas y debilidades de la organización empresarial mediante la cuantificación de todos sus recursos. Así mismo, precisa que la evaluación del sistema de control interno basado en principios, reglas, normas, procedimientos y sistemas de reconocido valor técnico es el fundamento de la realización de una buena auditoría.

La tesis elaborada por Alvarado \& Tuquiñahui(2011) "Propuesta de implantación de un sistema de Control interno basado en el modelo Coso" La Universidad Politécnica Salesiana, Aplicado a la Empresa Eléctrica Instalaciones en la ciudad de Cuenca, en la argumentación de la tesis propone diferentes metodologías empleadas para la administración de las empresas se ha visto la necesidad de impregnar en un concepto general al sistema de control interno aplicado a toda la organización. Este modelo nos permite analizar los diferentes componentes siendo este ambiente de control, evaluación de riesgos y actividades de control.

La tesis elaborada por Mendez (2010) "Evaluación de procedimientos de Control Interno en el área de cobros y créditos de una empresa distribuidora de Energía Eléctrica" La Universidad San Carlos de Guatemala aporta a las conceptualistas de esta variable, da a conocer los conceptos de auditoria así como la metodología que debe aplicarse en el proceso de evaluación de control interno .Desde el punto de vista de auditoria externa, se desarrolla la teoría del control interno de acuerdo al informe COSO.

Andrade (2012) elabora una propuesta de un sistema de control interno desarrollado para dar solución a las fallas que se presenten en la realización de las operaciones cuyo planteamiento del problema se originó en la observación directa de tareas y actividades que se cumplen.

El control interno debe ajustarse a las necesidades y requerimientos de cada organización debe consistir en un sistema que permita tener confianza moderada de que sus acciones administrativas se ajusten a los objetivos y normas legales estatutarias aplicables a la organización y exceso de control puede ser costoso y contraproducente.

\section{Conceptos importantes}

El control interno es el plan de organización y el conjunto de métodos y procedimientos que aseguren que los activos están debidamente protegidos, que los registros contables son fidedignos y que la actividad de la entidad se desarrolla eficazmente y se cumplen según las directrices mar- 
cadas por la Dirección." (Lara Bueno, 2007).

Con el objeto de proporcionar una garantía razonable para el logro de tres objetivos primordiales: 1) Obtención de información financiera correcta y segura; 2) Salvaguarda de activos; y, 3) Eficiencia y eficacia de las operaciones" (Rodríguez Valencia, 2006).

"La constitución política del Ecuador en su artículo 208 y 283, establece la obligatoriedad de la participación ciudadana en el control de recursos para promover el desarrollo e incentivar la producción nacional, la productividad y competitividad."

La Superintendencia de Compañías del Ecuador, ejerce un control sobre las empresas industriales, requiriendo informes de cumplimiento de objetivos, con niveles de eficiencia y efectividad.

Control Interno Proceso efectuado por la Dirección, la alta Gerencia y el resto del personal Proporcionar un grado de seguridad razonable en cuanto a la consecución de os objetivos, Eficacia, Eficiencia de las operaciones confiabilidad de la información financiera cumplimiento de las Leyes y normas establecidas.

Isaza (2012) En su libro Control Interno y Sistema de Gestión de Calidad en el capítulo 2, Modelo Estándar de Control Interno, para que nos competa, es la interdependencia que debe existir entre todos los componentes de una empresa. De control interno, obedece a los parámetros, estándares y/o controles que debe incluir una compañía en cada uno de sus procesos para prevenir errores y alcanzar condiciones estandarizadas de cantidad, calidad y costos.

Porter (2002) refiere que el éxito o el fracaso de cualquier empresa depende de su Ventaja Competitiva, entregar el producto a un costo menos le ofrece beneficios únicos para el comprador que justifiquen un precio superior. Pero ¿Cómo Lograr exactamente una compañía el liderazgo en costo? ¿Y cómo se diferencia de sus rivales?.

El ambiente de negocios actual cada vez más competitiva ha hecho ya de estrategia competitiva de Porter, una lectura obligada en escuelas de alta administración y uno de los libros mejor vendidos en cuanto a negocios de últimos anos Ventaja competitiva empieza donde acaba Estrategia Competitiva y proporciona guía específica para desarrollar salidas y poner en práctica.

Porter (1997) presenta una nueva dimensión en la planeación estratégica, subrayando la importancia de comprender la estructura del sector industrial o de negocio, así como la estructura de la competencia. Y provee a los directivos del material base para lograr que las fuerzas de mercado se inclinen a su favor... Porter hace una contribución sustancial a la estrategia descubriendo la riqueza de alternativas que todo director debe considerar.

\section{MATERIAL Y MÉTODOS}

El diseño de la investigación fue explicativo en el cual se observaron los fenómenos tal y como se dan en su contexto natural para después analizarlos y determinar la influencia de una variable sobre otra. En este trabajo investigativo, se orientó, determino y analizó el Control interno aplicado a la Empresa Eléctrica Regional del Sur S.A., de la ciudad de Loja y sus dos provincias Zamora Chinchipe y Morona Santiago y como se relaciona con la competitividad, esto nos permitió emitir juicios de valor acerca de cómo se encuentro en el sector eléctrico.
Unidad de análisis y población de estudio

Los objetos materia de estudio fueron las unidades orgánicas de la Empresa Eléctrica de la Región Sur S.A., del Ecuador, así como verificar su evaluación del sistema del control interno y situación de calidad y competitividad en el mercado eléctrico.

La población de la investigación, entendida a través de la Empresa Eléctrica Regional del Sur S.A., con área de 22,721 Km2 está compuesta por 16 cantones en Provincia de Loja, 9 Cantones en la Provincia de Zamora Chinchipe y 1 Cantón en la Provincia de Morona Santiago con un número de 400 empleados entre los que cuentan Directivos, Servidores Públicos y trabajadores a diciembre de 2014. (EERSSA, 2014)

\section{Tamaño de la Muestra}

Para definir el tamaño de la muestra se ha utilizado el siguiente método probabilístico.

$$
n=\frac{z^{2} x N \times P x Q}{E^{2} x(N-1)+Z^{2} x P x Q}
$$

Fuente: Ríos Delgado \& Torres Vázquez, Marzo 2013

Tabla 1.

Población de la encuesta / Fuente: (Ríos Delgado \& Torres Vázquez, Marzo 2013). Elaboración propia

\begin{tabular}{cc} 
Tamaño de la Población (N) & 400 \\
Error Muestra (E) & 0,05 \\
\hline Proporción de Éxito (P) & 0,5 \\
\hline Proporción de Fracaso (Q) & 0,5 \\
\hline Valor para Confianza (Z) (1) & 1,96 \\
\hline (1) Si: & $\mathrm{Z}$ \\
\hline Confianza el 99\% & 2,32 \\
\hline Confianza el 95\% & 1,96 \\
\hline Confianza el 92,5\% & 1,65 \\
\hline Confianza el 90\% & 1,28 \\
\hline
\end{tabular}


Tamaño de la muestra 196

Datos tomados en la Provincia de Loja, Zamora Chinchipe y Morona Santiago- Ecuador.

\section{Selección de la muestra}

Por la consideración de la naturaleza de la investigación fue necesario la muestra por cuanto fue conveniente entrevistar a cada miembro Directivo, Servidor Público y Trabajador de la EERSSA aplicando el método muestreo aleatorio simple, donde cada sujeto de la población tiene la misma probabilidad de ser seleccionado como dominado.

\section{RESULTADOS}

Los datos obtenidos, previamente seleccionados y registrados en los diferentes cuadros, como en las fichas bibliográficas, en cuestionarios y en medios electrónicos, fueron previamente seleccionados y depurados a través de las pruebas de validez y de confiabilidad; luego, ordenados y tabulados conforme a los indicadores de cada variable en los cuadros previamente preparados por la investigadora.

Tabla 2 .
Para la realización del trabajo y de la aplicación de los instrumentos, la encuesta y entrevista aplicada a la muestra seleccionada, cuyos resultados fueron debidamente procesados por medio del programa estadístico SPSS, y corroborado dichos resultados con la documentación a la cual se tuvo acceso producto de ello.

En cumplimiento con los objetivos propuestos se hace necesario mostrar las evidencias obtenidas de cada una de las conclusiones que permiten determinar las recomendaciones pertinentes, en tal sentido a continuación exponemos los resultados de la investigación.

\section{Prueba de Hipótesis}

Luego de probar uno a uno la distribución de los datos, de acuerdo a cada hipótesis propuesta, se procedió a elegir la probabilidad Exacta de Fisher, se tiene en cuenta los siguientes criterios:

1. La muestra es obtenida aleatoriamente.

2. Las variables se encuentran medidas nominalmente.
3. Más del $20 \%$ de las celdas que contienen las frecuencias esperadas de la tabla $3 \times 3$ tienen valores menores a cinco por lo que se deben unir las celdas adyacentes a estas celdas.

4. Al unir las celdas de la tabla $3 \times 3$, se obtuvo una tabla $2 \times 2$ la misma que presenta por lo menos una celda con valor esperado menor a cinco.

Entonces, la estadística de prueba a utilizar para contrastar las hipótesis se define de la siguiente manera:

$p(f)=\frac{(a+b) !(c+d) !(a+c) !(b+d) !}{n ! a ! b ! c ! d !}$

\section{Hipótesis A:}

Ho: El control interno no permite evaluar el cumplimiento de metas y objetivos en la empresa Eléctrica Regional del Sur S.A. del Ecuador.

H1: El control interno si, permite evaluar el cumplimiento de metas y objetivos en la empresa Eléctrica Regional del Sur S.A. del Ecuador.

Evaluación de cumplimiento de metas y objetivos de Hipótesis A Fuente: Elaboración propia

$\begin{array}{ccccc} & \text { PERMITE EVALUAR EL CUMPLIMIENTO DE METAS Y OBJETIVOS } & \text { Total } \\ \text { EL CONTROL INTERNO } & \mathrm{Si} & \text { No } & \text { Desconoce } & 126 \\ \mathrm{Si} & 101 & 101 & 0 & 29 \\ \mathrm{No} & 28 & 28 & 0 & 41 \\ \text { Desconoce } & 21 & 21 & 10 & \mathbf{1 0} \\ \text { Total } & \mathbf{1 5 0} & \mathbf{3 6} & \mathbf{1 0 6}\end{array}$

Para probar la hipótesis planteada seguiremos el siguiente procedimiento:

1.- Estadística de prueba: $p(f)=\frac{(a+b) !(c+d) !(a+c) !(b+d) !}{n ! a ! b ! c ! d !}$

3.- Cálculo de la estadística de prueba: Al desarrollar la fórmula tenemos:

2.- Nivel de significancia o error de tipo I: $\alpha=0,05$. 


$$
p(f)=\frac{(126) !(29) !(102) !(53) !}{196 ! 101: 25 ! 28 ! ! !}=1,4242 \mathrm{E}-106
$$

4.- Decisión estadística: Dado que

$1,42 \mathrm{E}-106<0,05$, se rechaza Ho.

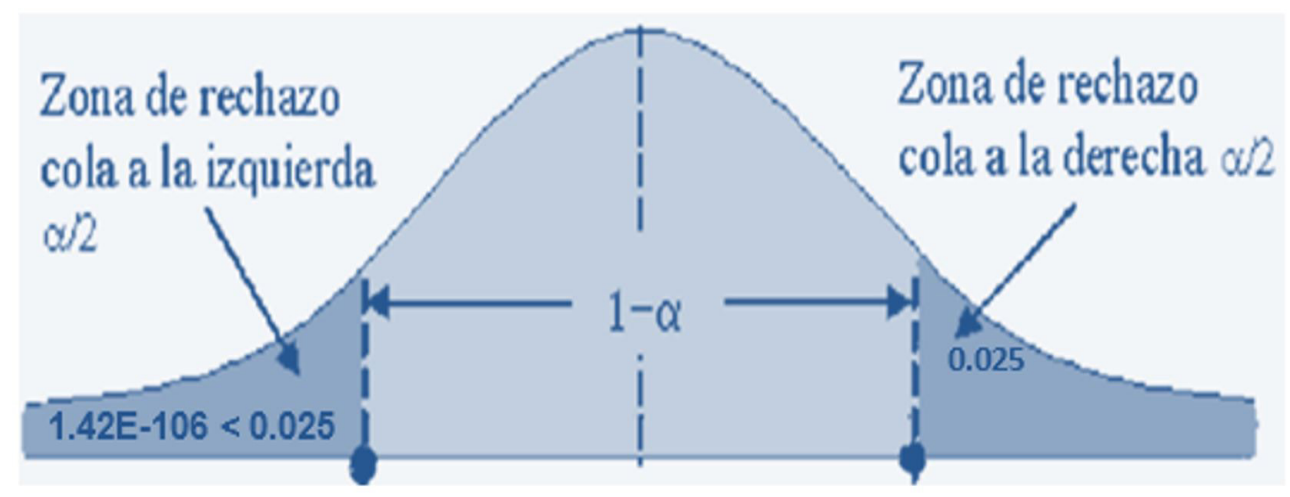

Figura 1. Hipótesis A

Fuente: Elaboración propia

6.- Conclusión: El control interno si permite evaluar el cumplimiento de metas y objetivos en la Empresa Eléctrica Regional del Sur S.A. del Ecuador.

\section{DISCUSIÓN}

Los datos e información obtenida como producto de la investigación determina, que el control interno es un proceso integral aplicado por la máxima autoridad y está orientado a cumplir con el ordenamiento jurídico, técnico y administrativo a evaluar el cumplimiento de metas y el logro de objetivos para garantizar la confiabilidad y oportunidad de la información para corregir las deficiencias del control en la Empresa Eléctrica Regional del Sur S.A. del Ecuador.

Del análisis a los datos e información procesada me permitió establecer que la evaluación de riesgos internos o externos es la identificación y posibilidad de ocurrencia de un evento no deseado que puede afectar a la entidad y permite analizar la cadena de valor como herramienta para la evaluación de la competitividad en la Empresa Eléctrica Regional del Sur S.A. del Ecuador.

Los datos obtenidos determinan que la actividad de control interno, si establece los controles adecuados para garantizar confiabilidad, seguridad y una administración clara de los niveles de acceso al sistema de información y comunicación eficaz con terceros como clientes, proveedores miembros de control la evaluación tecnológica de sistema automatizados que garanticen razonablemente la calidad de la información en la Empresa Eléctrica Regional del Sur S.A. del Ecuador.

Como producto de la contrastación de la hipótesis se ha determinado que la supervisión y seguimiento del control interno identifican y examinan brechas existentes frente a las expectativas relacionadas con las deficiencias en el sistema de control interno permitiendo mejorar la rentabilidad en la Empresa Eléctrica Regional del Sur S.A. del Ecuador.

Revisada la Situación Financiera de la EERSSA, en los periodos sujetos a revisión se establece que en los Activos y Pasivos de la Compañía se verificó la incidencia de estos resultados frente a la afectación del Patrimonio con lo cual se demuestra los EE.FF en el presente análisis:

Año 2012 al 2013 se incrementa 20\% equivalente a USD 23106215

Año 2013 al 2014 se incrementó 8\% equivalente a USD 9051187 Año 2014 al 2015 se incrementa 8,5\% equivalente a USD 11827566 
Concluyendo que recién en el año 2014 y 2015 se comienza a sanear cuentas que afectan la situación financiera y se las venía manteniendo sin ningún criterio técnico que determine la materialidad de la información.

En conclusión, se ha logrado determinar que la aplicación de los componentes del control interno si constituye un instrumento eficaz en el desarrollo de la competitividad empresarial ya que satisface al cliente en forma sostenida y rentable en el mercado eléctrico en sus tres provincias Loja, Zamora Chinchipe y Morona Santiago de la Empresa Eléctrica Regional del Sur S.A. del Ecuador periodo 2012-2014

\section{REFERENCIAS BIBLIOGRAFICAS}

Alvarado, M. A., \& Tuquiñahui, S. P. (2011). Propuesta de implementación de un sistema de control interno basado en el modelo Coso, aplicado a la em- presa electro instalaciones en la ciudad de Cuenca. Universidad Politécnica Salesiana: Cuenca.

Andrade, L. (2012). Diseño de un sistema de control interno administrativo, financiero y contable aplicable a la empresa CEDETEC S.A. Universidad Politécnica Salesiana: Quito.

EERSSA, J. d. (2014). Servicios por Tarifas y Cantones a Diciembre de 2014. Loja - Ecuador.

Gómez, E. G. (2001). Introducción a la evaluación del control interno. Revista Indexada. De: https://www.gestiopolis.com/introduccion-evaluacion-sistema-control-interno/

Isaza, A. T. (2012). Control Interno y Sistema de Calidad. Bogotá: Ediciones de la U.

Lara Bueno, M. I. (2007). Manual básico de revisión y verificación contable. Madrid: Dykison.
Mendez, E. (2010). Evaluación de procedimientos de Control Interno en el área de cobros y créditos de una empresa distribudora de energía eléctrica. Universidad San Carlos de Guatemala: Guatemala.

Porter, M. (1997). Estrategia Competitiva. Ciudad de México: Continental S.A. de C.V.

Porter, M. (2002). Ventaja competitiva. México D.F: SL Grupo patria cultural- Alay Ediciones.

Ríos Delgado, T. J., \& Torres Vázquez, C. P. (2013). La Tesis Teoría y Práctica. Lima: Editorial de la Universidad Nacional Mayor de San Marcos.

Rodríguez Valencia, J. (2006). Control Interno: Un efectivo sistema para la empresa. Colombia: Editorial Trilla. 